Bull. Austral. Math. Soc.

20E $10,20 \mathrm{~F} 06$

Vol. 51 (1995) [287-290]

\title{
ON THE JOIN OF VARIETIES OF GROUPS
}

\author{
A. StorozheV
}

We introduce two pseudoabelian group varieties, each defined by one word in two variables, and prove that their join is not pseudoabelian. We also prove that every variety which is nilpotent of class two, is not join-inaccessible.

In most cases, given the sets of laws for two group varieties, it is very difficult to obtain a description of the set of laws for the join of the varieties. Therefore it is important to find connections between properties of varieties and of their join. In 1982, Kovács raised the following question [1, problem 8.22]. If $G$ is a finite group contained in a join $\mathfrak{U} \vee \mathfrak{V}$ of two varieties $\mathfrak{U}, \mathfrak{V}$ of groups, must there exist finite groups $A \in \mathfrak{U}, B \in \mathfrak{V}$ such that $G$ is a section of the direct product $A \times B$ ? In [3] a variety is called pseudoabelian if it is nonabelian but all its finite groups are abelian. Answering a question of $H$. Neumann [4, problem 5], Ol'shanskii [5] proved the existence of pseudoabelian varieties of groups. In 1973, Kovács and Newman [3] stated a question concerning the join of two pseudoabelian varieties: must the join of two pseudoabelian varieties be pseudoabelian? According to [2], a variety $\mathfrak{U}$ is called join-inaccessible if $\mathfrak{U} \subset \mathfrak{X} \vee \mathfrak{Y}$ implies $\mathfrak{U} \subset \mathfrak{X}$ or $\mathfrak{U} \subset \mathfrak{Y}$. In [2] Kovács raised the question as to whether the nilpotent just-nonabelian varieties $\left(\mathfrak{B}_{4} \wedge \mathfrak{N}_{2}\right.$ and $\mathfrak{B}_{p} \wedge \mathfrak{N}_{2}$ for odd primes $\left.p\right)$ are join-inaccessible.

In this paper we prove that there exist two pseudoabelian group varieties such that their join is not pseudoabelian and we also show that every variety which is nilpotent of class two, is not join-inaccessible. These results imply negative answers to all three questions cited above.

Received 19th May, 1994.

This work was completed while the author was on a visit to the University of Queensland, supported by an ARC grant.

Copyright Clearance Centre, Inc. Serial-fee code: $0004-9729 / 95 \quad \$ A 2.00+0.00$. 
We put

$$
\begin{gathered}
v_{0}=v_{0}(x, y)=\left(x^{d} y^{d}\right)^{d} x^{d}, \\
v_{1}=v_{1}(x, y)=\left[v_{0}^{d}, x^{d}\right], \\
v_{2}=v_{2}(x, y)=\left[v_{1}^{d}, x^{d}\right], \\
v_{3}=v_{3}(x, y)=\left[v_{2}^{d}, x^{d}\right], \\
w_{1}(x, y)=[x, y] v_{2}^{n}[x, y]^{e_{1}} v_{2}^{n+1} \cdots[x, y]^{e_{h}} v_{2}^{n+h}, \\
w_{2}(x, y)=[x, y] v_{3}^{n}[x, y]^{e_{1}} v_{3}^{n+1} \cdots[x, y]^{e_{h}} v_{3}^{n+h},
\end{gathered}
$$

where $h \equiv 0(\bmod 10), \varepsilon_{10 k+1}=\varepsilon_{10 k+2}=\varepsilon_{10 k+3}=\varepsilon_{10 k+5}=\varepsilon_{10 k+6}=1, \varepsilon_{10 k+4}=$ $\varepsilon_{10 k+7}=\varepsilon_{10 k+8}=\varepsilon_{10 k+9}=\varepsilon_{10 k+10}=-1, k=0,1, \cdots, h / 10-1$ and $d, n, h$ are sufficiently large natural numbers.

Let $W_{i}$ denote the verbal subgroup of an absolutely free group $F$ of countable rank corresponding to the word $w_{i}(x, y)$ and let $\mathfrak{M}_{i}$ be the variety defined by the word $\boldsymbol{w}_{\boldsymbol{i}}(\boldsymbol{x}, \boldsymbol{y}), i=1,2$. Besides the varieties $\mathfrak{M}_{1}, \mathfrak{M}_{2}, \mathfrak{M}_{1} \vee \mathfrak{M}_{2}, \mathfrak{M}_{1} \wedge \mathfrak{M}_{2}$, we need to consider the variety $\left[\mathfrak{O}, \mathfrak{M}_{1} \wedge \mathfrak{M}_{2}\right]$ which is defined by the set of words $\left[F, W_{1} W_{2}\right]$ and of which a free group is the free central extension of a free group of the variety $\mathfrak{M}_{1} \wedge \mathfrak{M}_{2}$.

The construction and the study of the varieties $\mathfrak{M}_{1}, \mathfrak{M}_{2}, \mathfrak{M}_{1} \wedge \mathfrak{M}_{2}$ and $\left[\mathfrak{O}, \mathfrak{M}_{1} \wedge \mathfrak{M}_{2}\right]$ follow the patterns detailed in $[6,25.1],[6,29.3]$ and [7]. In particular, the relatively free groups $G_{1}=F / W_{1}, G_{2}=F / W_{2}, G=F / W_{1} W_{2}$ are described in terms of their presentations with defining relations being introduced by induction.

LеммA 1. The varieties $\mathfrak{M}_{1}$ and $\mathfrak{M}_{2}$ are pseudoabelian varieties.

Proof: When substituting $[x, z]$ for every $x$ in the word $w_{i}(x, y), i=1,2$, and taking into account the equation $\varepsilon_{1}+\ldots+\varepsilon_{h}=0$, we see that every metabelian group of the variety $\mathfrak{M}_{i}$ is nilpotent of class two. But it is also easily seen that in the variety $\mathfrak{M}_{i}$ all groups nilpotent of class two are abelian. So all metabelian groups in the varieties $\mathfrak{M}_{1}$ and $\mathfrak{M}_{2}$ are abelian. Hence all finite groups of the varieties $\mathfrak{M}_{1}$ and $\mathfrak{M}_{2}$ are abelian. The fact that the varieties $\mathfrak{M}_{1}$ and $\mathfrak{M}_{2}$ are nonabelian is proved in the same way as the corresponding part of [6, Theorem 30.1].

Now we consider the presentation $G=\langle\mathfrak{A} \mid R=1, R \in \mathfrak{R}\rangle$ chosen in respect to the prescriptions of $[6,29.3]$ and $[7]$ where $\mathfrak{A}$ is a basis of the group $F$.

LEmma 2. The presentation $G=G(\infty)=\langle\mathfrak{A} \mid R=1, R \in \mathfrak{R}\rangle$ satisfies condition $R$.

Lemma 2 is proved as Lemma 8 [7].

LEMmA 3. Nontrivial values of the words $v_{2}(x, y)$ and $v_{3}(x, y)$ in $G$ cannot be conjugate in $G$ to powers of the same word that is a simple word or a period of the presentation $G(\infty)$. 
Lemma 3 can be proved in the same way as the corresponding parts of $[7$, Lemma 5] and [7, Lemma 10].

LEMma 4. Nontrivial values $w_{1}\left(X_{1}, Y_{1}\right)$ and $w_{2}\left(X_{2}, Y_{2}\right)$ cannot be equal in $\widetilde{G}=$ $F /\left[F, W_{1} W_{2}\right]$.

Proof: Suppose that $w_{1}\left(X_{1}, Y_{1}\right)=w_{2}\left(X_{2}, Y_{2}\right)$ in $\tilde{G}$ where $\left(X_{1}, Y_{1}\right)$ and $\left(X_{2}, Y_{2}\right)$ are two pairs of words noncommutative in $G$. As in the proof of [6, Lemma 39.12], we can find defining words $R_{1}$ and $R_{2}$ which are conjugate to $w_{1}\left(X_{1}, Y_{1}\right)$ and $w_{2}\left(X_{2}, Y_{2}\right)$ respectively in rank less than the maximum of the ranks of $R_{1}$ and $R_{2}$. So there exists an annular diagram $\Delta$ such that the labels of its contours are graphically equal to $R_{1}$ and $R_{2}^{ \pm 1}$ and the number of cells of ranks not less than the maximum of the ranks of $R_{1}$ and $R_{2}$ is even in $\Delta$. Since $R_{1} \not \equiv R_{2}$ by Lemma 3 , the existence of the diagram $\Delta$ contradicts Lemma 2 and [6, Lemma 25.1].

THEOREM . $\mathfrak{M}_{1} \vee \mathfrak{M}_{2} \supset\left[\mathfrak{O}, \mathfrak{M}_{1} \wedge \mathfrak{M}_{2}\right]$.

Proof: Given a word $U \in W_{1} \cap W_{2}$, we may consider equations

$$
w_{1}\left(X_{1}, Y_{1}\right) w_{1}\left(X_{2}, Y_{2}\right) \ldots=U=w_{2}\left(X_{1}^{\prime}, Y_{1}^{\prime}\right) w_{2}\left(X_{2}^{\prime}, Y_{2}^{\prime}\right) \ldots
$$

in $\widetilde{G}$. In the same way as in the proof of [6, Lemma 39.16] we obtain that all nontrivial values of the words $w_{1}(x, y)$ and $w_{2}(x, y)$ in $\tilde{G}$ form a basis for the free abelian group $W_{1} W_{2} /\left[F, W_{1} W_{2}\right]$. By Lemma 4, nontrivial values $w_{1}\left(X_{i}, Y_{i}\right)$ and $w_{2}\left(X_{j}^{\prime}, Y_{j}^{\prime}\right)$ are different in $\tilde{G}$. Hence $U=1$ in $\tilde{G}$ whence $W_{1} \cap W_{2} \subset\left[F, W_{1} W_{2}\right]$.

Corollary 1. The join $\mathfrak{M}_{1} \vee \mathfrak{M}_{2}$ of two pseudoabelian varieties $\mathfrak{M}_{1}$ and $\mathfrak{M}_{2}$ is not pseudoabelian.

Proof: Any nonabelian finite group that is nilpotent of class two belongs to the variety $\mathfrak{M}_{1} \vee \mathfrak{M}_{2}$ by the Theorem. Thus the variety $\mathfrak{M}_{1} \vee \mathfrak{M}_{2}$ is not pseudoabelian but $\mathfrak{M}_{1}$ and $\mathfrak{M}_{2}$ are both pseudoabelian by Lemma 1 .

Corollary 2. Every variety which is nilpotent of class two, is not joininaccessible.

Proof: Any variety that is nilpotent of class two is a subvariety of $\mathfrak{M}_{1} \vee \mathfrak{M}_{2}$ by the Theorem. But as was noted in the proof of Lemma 1, every group, which is nilpotent of class two and belongs to $\mathfrak{M}_{1}$ or $\mathfrak{M}_{2}$, is abelian. Therefore, no nilpotent nonabelian variety is a subvariety of $\mathfrak{M}_{1}$ or $\mathfrak{M}_{2}$.

\section{REFERENCES}

[1] Kourovka Notebook, Unsolved problems of the group theory, Tenth Edition (Novosibirsk, 1986). 
[2] L.G. Kovács, 'Inaccessible varieties of groups', Bull. Austral. Math. Soc. 17 (1974), 506-510.

[3] L.G. Kovács and M.F. Newman, 'Hanna Neumann's problems on varieties of groups', in Proceedings of the Second International Conference on the Theory of Groups, Lecture Notes in Mathematics 372 (Springer-Verlag, Berlin, Heidelberg, New York, 1974), pp. 417-431.

[4] H. Neumann, Varieties of groups (Springer-Verlag, Berlin, Heidelberg, New York, 1967).

[5] A.Yu. Ol'shanskii, 'Varieties in which all finite groups are abelian', Mat. Sb. 126 (1985), 59-82.

[6] A.Yu. Ol'shanskii, Geometry of defining relations in groups, Mathematics and its application (Soviet Series) 70 (Kluwer Academic Publishers, Dordrecht, 1991).

[7] A. Storozhev, 'On abelian subgroups of relatively free groups', Comm. Algebra 22 (1994), 2677-2701.

Australian Mathematics Trust

University of Canberra

Belconnen ACT 2616

Australia 